\section{Yeast Sm-like proteins function in mRNA decapping and decay}

\author{
Sundaresan Tharun ${ }^{\star} \dagger$, Weihai He ${ }^{\star} \dagger$, Andrew E. Mayes $\neq \$$, \\ Pascal Lennertz ${ }^{\star}$, Jean D. Beggs $\ddagger$ \& Roy Parker ${ }^{\star}$ \\ ${ }^{*}$ Department of Molecular and Cellular Biology and Howard Hughes Medical \\ Institute, University of Arizona, Tucson, Arizona 85721, USA \\ \$Institute of Cell and Molecular Biology, University of Edinburgh, \\ King's Buildings, Mayfield Road, Edinburgh EH9 3JR, UK \\ $\$$ Unilever Research Colworth, Colworth House, Sharnbrook, Bedford, \\ MK44 1LQ, UK \\ + These authors contributed equally to this work
}

One of the main mechanisms of messenger RNA degradation in eukaryotes occurs by deadenylation-dependent decapping which leads to $5^{\prime}$-to-3' decay ${ }^{1,2}$. A family of Sm-like (Lsm) proteins has been identified, members of which contain the ' $\mathrm{Sm}$ ' sequence motif, form a complex with U6 small nuclear RNA and are required for pre-mRNA splicing ${ }^{3-9}$. Here we show that mutations in seven yeast Lsm proteins (Lsm1-Lsm7) also lead to inhibition of mRNA decapping. In addition, the Lsm1-Lsm7 proteins coimmunoprecipitate with the mRNA decapping enzyme (Dcp1), a decapping activator (Pat1/Mrt1) and with mRNA. This indicates that the Lsm proteins may promote decapping by interactions with the mRNA and the decapping machinery. In addition, the Lsm complex that functions in mRNA decay appears to be distinct from the U6-associated Lsm complex, indicating that Lsm proteins form specific complexes that affect different aspects of mRNA metabolism.

We proposed that the family of Lsm proteins affects mRNA degradation because $l s m 1$ strains are deficient in mRNA decapping ${ }^{10}$ and the Lsm proteins interact with each other ${ }^{6,7}$. Thus, we examined the decay of the $M F A 2 p G$ and $P G K 1 p G$ reporter mRNAs ${ }^{11}$ in strains with the nonessential LSM1, LSM6 or LSM7 genes (each of which is thermosensitive) deleted, and in strains carrying temperaturesensitive forms of LSM2 or LSM5, or a partial loss-of-function allele of LSM8. The reporter mRNAs are under the control of the GAL1 UAS to allow measurement of their decay rates and contain a poly $(\mathrm{G})$ tract in their $3^{\prime}$ untranslated region (UTR), which traps decay intermediates by blocking the $5^{\prime}$-to- $3^{\prime}$ exonuclease in $c i s^{12,13}$. Mutations inhibiting mRNA turnover accumulate full-length mRNA and show a decrease in the levels of the decay intermediate ${ }^{12}$.

Two observations indicated that loss-of-function alleles of LSM1-LSM7 inhibited mRNA degradation. First, loss-of-function alleles in the LSM1, LSM2, LSM5, LSM6 and LSM7 genes led to increased levels of full-length mRNA and decreased levels of decay intermediate for both the $M F A 2 p G$ (Fig. 1, in $0 \mathrm{~min}$ ) and $P G K 1 p G$ (data not shown) transcripts. Second, the $M F A 2 p G$ transcript showed a slower decay rate (Fig. 1) in the $l \operatorname{sm} 1, l \operatorname{sm} 2, l \operatorname{sm} 5, l \operatorname{sm} 6$ and $l s m 7$ mutant strains as compared with wild-type strains. Although the $l s m 1, l s m 2, l s m 5, l s m 6$ and $l s m 7$ alleles cause thermosensitive growth, we observed the effects on mRNA decay at both $24^{\circ} \mathrm{C}$ (Fig. 1) and $37^{\circ} \mathrm{C}$ (data not shown). The lsm8-1 allele examined did not affect mRNA turnover. The inactivation of the Lsm proteins caused a partial defect in mRNA decay as compared with the complete block seen in $d c p 1 \Delta$ and $d c p 2 \Delta$ strains $^{14,15}$, suggesting that Lsm proteins activate mRNA decay. Consistent with this view, an $l \operatorname{sm} 1 \Delta l \sin 6 \Delta$ double mutant strain did not show an increased defect in mRNA decay (Fig. 1).

To determine which step in mRNA degradation was affected in the $l s m$ mutants, we examined the structure of the MFA2pG mRNA in these strains. Strains deficient in decapping or in $5^{\prime}$-to- $3^{\prime}$ exonucleolysis accumulate oligoadenylated, full-length mRNA species, with or without cap, respectively ${ }^{10,12,14-16}$. Thus, we examined the poly(A) distribution on the MFA2pG mRNA in the lsm mutants with or without fractionation into capped and uncapped fractions by immunoprecipitation with anti-cap antibodies. Strains mutant in LSM1-LSM7 accumulated MFA2pG transcripts with short poly(A) tails (Fig. 2, T lanes), indicating that these strains were deficient in decapping or in $5^{\prime}$-to- $3^{\prime}$ exonucleolysis. A substantial amount of the accumulated oligoadenylated species in the $l s m 1, l s m 2, l s m 5, l s m 6$ and $l s m 7$ strains was immunoprecipitated by antisera against the cap structure, similar to the $d c p 1 \Delta$ strain, wherein decapping is blocked (Fig. 2). This indicated that LSM2, LSM5, LSM6 and LSM7 are required, as is LSM1 (ref. 10) for efficient mRNA decapping. Moreover, in strains where the only copy of the essential LSM3 or LSM4 is expressed from a GAL promoter, transfer of the culture to glucose medium (thereby depleting Lsm3 or Lsm4 (ref. 7) led to the accumulation of capped, deadenylated MFA2pG mRNAs (Fig. 2). This observation, together with the co-immunoprecipitation of Lsm3 and Lsm4 with mRNA decay factors (see below), suggests that LSM3 and LSM4 are also required for efficient mRNA decapping.

Two-hybrid analysis has identified interactions between Lsm proteins and the Dcp1 decapping enzyme, the Xrn1 5'-to-3' exonuclease and Dcp2 (ref. 17). The Lsm proteins also show

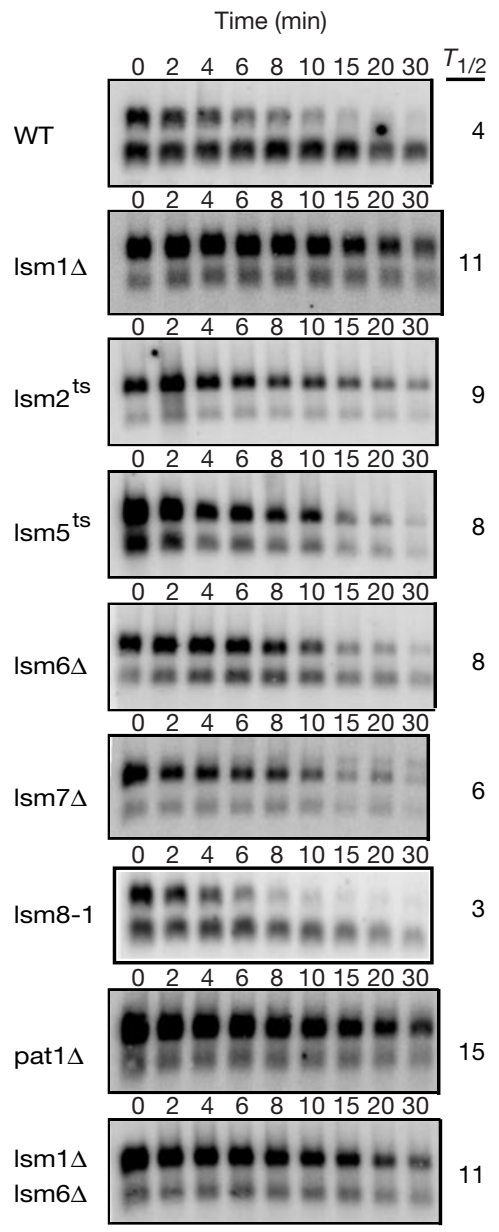

Figure 1 MFA2pG mRNA is stabilized in most Ism mutant strains. The decay of the MFA2p G transcript after repression of transcription by the addition of glucose in $1 \mathrm{sm}$ mutants and wild-type (WT) strain at $24^{\circ} \mathrm{C}$ is shown. The top band is the full-length mRNA and the lower band is the mRNA fragment produced by $5^{\prime}$-to-3' exonucleolytic digestion to the $5^{\prime}$ side of the poly(G) tract ${ }^{11}$. The strains used were WT (yRP841), Ism1 $1 \Delta$ (yRP1365), Ism2 $2^{\text {ts }}$ (yRP1366), Ism5 ${ }^{\text {ts }}$ (yRP1367), Ism6 (yRP1369), Ism7 (yRP1370),

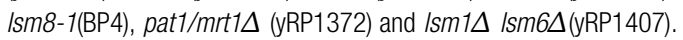


two-hybrid interactions with Pat1 (YCR077c) ${ }^{17}$, a protein originally identified through a two-hybrid interaction with topoisomerase II (ref. 18). Pat1 $\Delta$ strains have multiple phenotypes, including temperature sensitivity, that are similar to mutations in the MRT1 gene, an uncloned locus known to affect mRNA decapping ${ }^{12}$. There is evidence that MRT1 and PAT1 are the same gene. First, the PAT1 gene complements the temperature sensitivity and mRNA decay defect in $m r t 1$ strains (data not shown). Second, pat1 $\Delta$ strains show a defect in mRNA decay similar to the mrt1 alleles (Fig. 1). Third, sequencing of the PAT1 gene from strains carrying the mrt1-1, mrt12 and mrt $1-3$ alleles identified mutations creating nonsense codons at amino acids 547, 157 and 308, respectively. Fourth, the mrt1 alleles and the pat $1 \Delta$ are genetically linked and pat1 $\Delta$ fails to complement the mrt1 lesions in diploids (data not shown). Thus, PAT1/MRT1 is required for efficient mRNA decapping.

To investigate the significance of the two-hybrid interactions between the Lsm proteins and the proteins required for mRNA decapping, we immunoprecipitated epitope-tagged proteins Lsm1-
Lsm8 and determined whether Dcp1 or Pat1/Mrt1 co-immunoprecipitated by using western analysis. Dcp1 co-immunoprecipitated with Lsm1-Lsm7, although the efficiency of coimmunoprecipitation varied (Fig. 3a). No co-immunoprecipitation of Dcp1 with Lsm8 could be detected, however, even after long exposures. Similarly, Pat1/Mrt1 also co-immunoprecipitates with Lsm1-Lsm7 proteins, but not with Lsm8 (Fig. 3b; and data not shown).

The Lsm and Sm proteins are RNA-binding complexes and function in splicing by assembling with snRNAs ${ }^{3,4}$. To investigate whether Lsm proteins affect decapping through interactions with mRNA, we determined whether mRNAs co-immunoprecipitated with Lsm proteins (Fig. 4). Lsm1, Lsm5 and Dcp1 co-immunoprecipitated with a small (1-3\% of the total) but clearly detectable amount of $\mathrm{CYH} 2 \mathrm{mRNA}$. In contrast, Lsm8 did not co-immunoprecipitate the $\mathrm{CYH} 2 \mathrm{mRNA}$ in amounts significantly above background levels. Moreover, we did not detect U6 snRNA in immunoprecipitates of Lsm1 and Dcp1, which indicates that

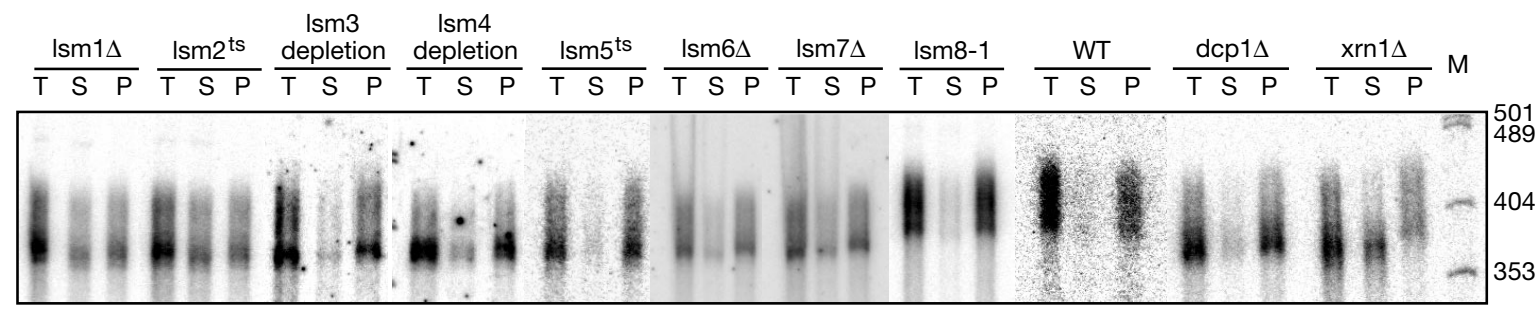

Figure 2 Lsm mutants accumulate oligoadenylated, full-length capped mRNAs. Steadystate mRNA from various $I s m$ mutants, $d c p 1 \Delta, x r n 1 \Delta$ and wild-type strains were analysed on a polyacrylamide northern blot with or without immunoprecipitation with anticap antibodies. Strains expressing LSM3 and LSM4 under GAL control were shifted to

glucose for $12 \mathrm{~h}$ before analysis, which has been shown to deplete these proteins ${ }^{7} . T$, total RNA; P, RNA extracted from the immunoprecipitate; S, supernatant. Radiolabelled Hpalldigested pUC18 DNA was used as marker (M).

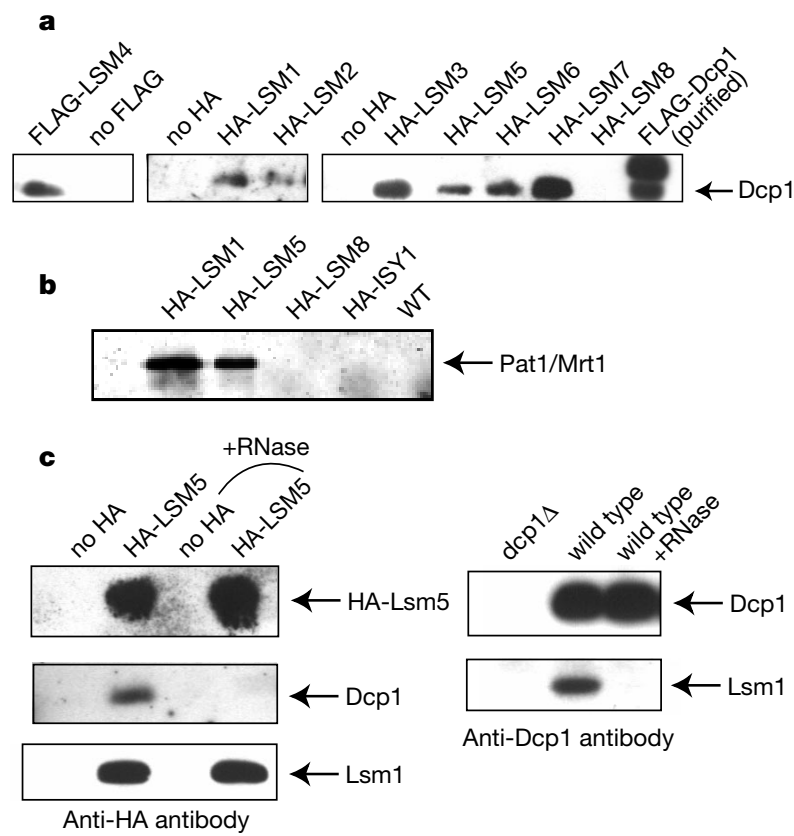

Figure $3 \mathrm{Lsm}$ proteins co-immunoprecipitate with proteins involved in mRNA decay. a, Dcp1 co-immunoprecipitates with proteins Lsm1-Lsm7. Epitope-tagged Lsm proteins were immunoprecipitated and the immunoprecipitates were analysed by western blot for Dcp1. b, Co-immunoprecipitation of Pat1 with HA-tagged Lsm proteins. Epitope-tagged Lsm proteins were immunoprecipitated and the immunoprecipitates were analysed by western blot for Pat1. Isy1, a protein involved in pre-mRNA splicing ${ }^{22}$, was used as a control. c, Interaction between Dcp1 and Lsm proteins is RNA dependent. Immunoprecipitation of Dcp1 (right panel) and HA-Lsm5 (left panel) from lysates of different strains (indicated above the lanes) was done with ('+RNase') or without prior RNase treatment and presence of co-immunoprecipitating proteins (Dcp1 and Lsm1) was analysed by western blot using polyclonal Dcp1 or Lsm1 antisera. 
these interactions are RNA specific (Fig. 4b; and data not shown). These results suggest that Lsm proteins interact with mRNA molecules and thereby modulate decapping. The co-immunoprecipitation of Lsm proteins with Dcp1, but not with each other or Pat1, is sensitive to RNase treatment (Fig. 3c and data not shown). This suggests that either the Lsm complex and Dcp1 interact directly in a manner dependent on one or both of these proteins binding mRNA, or that these proteins interact simultaneously with the same mRNA molecules. Thus, the co-immunoprecipitation of Lsm proteins, Dcp1 and mRNA may reflect the detection of a transient mRNP (ribonuclear protein particle) organization that exists before mRNA decapping.

These physical interactions suggest that the Lsm proteins have a role in cytoplasmic mRNA degradation. Consistent with this view, Lsm1, Lsm7, Dcp1 and Pat1 can be detected in the cytoplasm by immunofluorescence (Fig. 5). Lsm7 was also found in both compartments, consistent with it affecting both mRNA decay and splicing ${ }^{6,7}$. Some nuclear staining was observed for both Dcp1 and Lsm1, suggesting that these proteins may also have a nuclear function.

Several observations indicate that a complex, consisting of Lsm1Lsm7, is involved in mRNA decay and that a different complex, consisting of Lsm2-Lsm8, is associated with U6 and involved in pre-mRNA splicing. First, although mutations in LSM2-LSM7 affect both mRNA decay and pre-mRNA splicing, mutations in LSM1 only affect mRNA decay, and lesions in LSM8 affect splicing but not mRNA decay (Fig. 1) ${ }^{7,8,10}$. Second, proteins Lsm2-Lsm7 coimmunoprecipitate with both U6 snRNA ${ }^{6,7,9}$ and mRNA decay factors (Fig. 3), whereas Lsm1 does not immunoprecipitate U6 snRNA $^{6,7}$ but does pull down Dcp1 and Pat1/Mrt1 (Fig. 3). In contrast, Lsm8 does co-immunoprecipitate U6 snRNA ${ }^{6-9}$ but does not pull down Dcp1 or Pat1/Mrt1 (Fig. 3). Third, immunoprecipitation of Lsm8 co-immunoprecipitates Lsm2-Lsm7, but not Lsm1 (ref. 6). Fourth, a pair of distinct complexes, each consisting of seven members, would be consistent with the known organization of Sm and Lsm proteins ${ }^{19}$. This suggests that the Lsm proteins form at least two distinct complexes that are involved in different aspects of RNA metabolism.

The function of the Lsm proteins in decapping appears to be to interact with the mRNA substrate and accelerate decapping, perhaps by promoting rearrangements in $\mathrm{mRNP}$ organization that

a
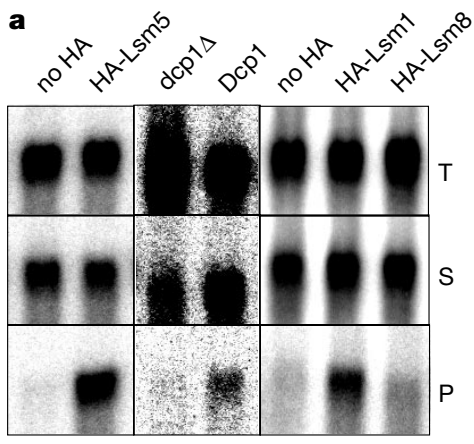

CYH2 mRNA

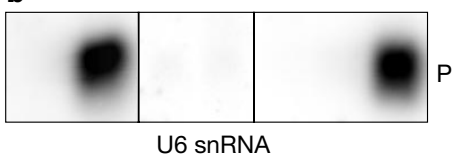

Figure $4 \mathrm{Lsm}$ proteins and Dcp1 co-immunoprecipitate mRNA. RNA was extracted from the immunoprecipitate $(\mathrm{P})$ of Dcp1, HA-Lsm5, HA-Lsm1 or HA-Lsm8 and analysed by northern blot for $\mathrm{CYH} 2 \mathrm{mRNA}$ (a) or U6 snRNA (b). Similar analysis of aliquots of the lysate (T), and supernate (S) for the CYH2 mRNA is also shown (a).

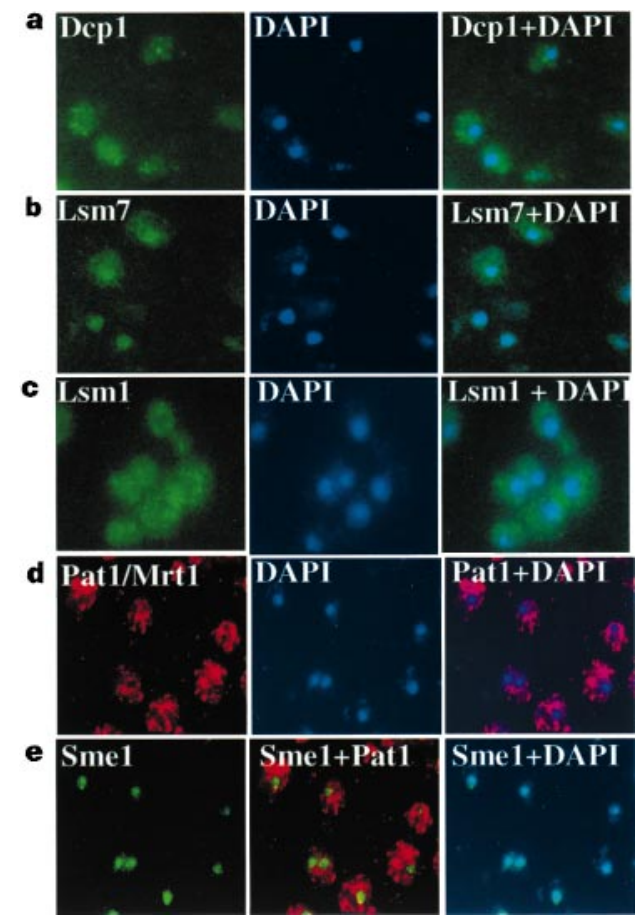

Figure 5 Cellular localization of Dcp1, Pat1/Mrt1, Lsm7 and Lsm1 by immunofluorescence analysis. a-c, Strains expressing HA-tagged Dcp1 (AEMY121 (a)), Lsm7 (AEMY51 (b)) or Lsm1 (AEMY28 (c)) were stained with anti-HA antibodies and anti-rat (fluorescein isothiocyanate) secondary antibody (green fluorescence, left panel), or subjected to 4,6-diamidino-2-phenylindole (DAPI) nuclear staining (blue fluorescence, middle panel). A merger of the two panels is shown in the right panel in each case. d, AEMY54 cells were stained with anti-Pat1 antibodies and anti-rabbit (cy3) secondary antibody (red fluorescence, left panel), or DAPI nuclear stain (blue fluorescence, middle panel). e, As a control, cells expressing the HA-tagged Sme1 nuclear protein (AEMY54) were stained with anti-HA antibodies and anti-rat (fluorescein isothiocyanate) secondary antibody (green fluorescence, left panel). A merge of $\mathrm{HA}-\mathrm{Sme} 1$ staining and Pat1 staining (middle panel), and a merge of HA-Sme1 staining and DAPI nuclear staining (right panel).

lead to dissociation of the cytoplasmic cap-binding complex, which is an inhibitor of decapping ${ }^{20}$. A general role for Lsm proteins in modulating RNP structure in a variety of contexts by affecting RNA-RNA and/or protein-RNA interactions would be consistent with the proposal that their function in pre-mRNA splicing is to facilitate RNP rearrangements during splicing ${ }^{7,21}$.

\section{Methods}

\section{Yeast strains and plasmids}

All strains used for decay analysis were in the genetic background of yRP841 (MAT $\alpha$ trp1 ura3-52 leu2-3,112 lys2-201 cup1 $\Delta:: L E U 2(P M))^{12}$, except the lsm8-1 allele, which was compared with an isogenic wild-type control. Each strain and its genotypic difference from yRP841 are as follows: yRP1365 (lsm1 $\Delta::$ TRP1); yRP1366 (lsm2 $\Delta:: H I S 3$ pAEM55 (carrying a protein fusion that confers temperature sensitivity to Lsm2); yRP1367 (his4-539 lsm5 $\Delta:: T R P 1$ pRP949 (carrying a protein fusion that confers temperature sensitivity to Lsm5); yRP1369 (lsm6 $::$ HIS3); yRP1370 (lsm74::HIS3 LYS2); yRP1372 (MATa his4-539 pat1 $\Delta:: L E U 2) ; y R P 1407$ (MATa lsm1 $\Delta:: T R P 1$ lsm6 $:: H I S 3) ; y R P 1070$ $(d c p 1 \Delta:: U R A 3)^{14}$; yRP884 (xrn1 $\left.\Delta:: U R A 3\right)^{11}$; BP4 (ref. 8) (MATa lsm8-1) and its isogenic wild-type control CY3 (ref. 8) (MATa ura3 lys2 ade2 trp1 his3 leu2). All strains used for immunoprecipitation, except FLAG-LSM4 strain, were in the same genetic background, expressing an haemagglutin A (HA)-tagged Lsm protein and compared with isogenic wild-type strains lacking any epitope tags (BMA64) ${ }^{7}$. Each strain and its genotypic difference from BMA64 (ref. 7) (MAT $\alpha$ trp1 $\Delta 1$, his3-11, -15, ura3-1, leu2-3, -112, ade2-1, can-100) are as follows: AEMY30 (trp1-1 his3 200 lsm2 $2:: H I S 3$ pAEM55 (HA-LSM2); AEMY33 (ref. 7) (trp1-1 his3 200 lsm2 $\Delta:: H I S 3$ pAEM55 (HA-LSM2); AEMY33 (ref. 7) (lsm3 $\triangle::$ TRP1 pAEM64 (GAL-HA-LSM3)); AEMY28 (ref. 7 (HA-LSM1); AEMY45 (Mata lsm8 $\Delta:: T R P 1$ pAEM76 (GAL-HA-LSM8)); AEMY47 (ref. 7) (lsm5 $:: T R P 1$ pAEM75 (GAL-HA-LSM5)). AEMY49 (MATa LSM6-HA-TRP1), AEMY50 (MATa 
LSM7-HA-TRP1); IDY2 (ref. 22) (MATa trp1-1 his3 200 isy1 $:: H I S 3$ pCA23 (HA-ISY1, CEN URA3)). For Lsm4, the MCY4 (ref. 5) strain expressing GPD promoter-controlled FLAG-Lsm4 protein from a plasmid (pRP961) was used. This strain has a chromosoma LSM4 gene under GAL UAS control and was grown in glucose medium for the experiment. MCY4 (ref. 5) (MATa LEU2-GAL-LSM4) was compared with its isogenic wild-type strain w303 (MAT $\alpha$ ade1-101 his3- $\Delta 1$ trp1-289 ura33-52) without epitope-tagged proteins.

\section{RNA analysis}

Transcriptional shut-off experiments, northern analysis and immunoprecipitation of RNA were performed as described ${ }^{12,13,15}$. Oligo oRP140 (5' ${ }^{\prime}$-ATATTGATTAGATCAGGAATTCC-3') was used to probe for MFA2pG mRNA.

\section{Immunology}

The immunoprecipitation and immunofluorescence experiments followed standard protocols. The HA-Lsm protein immunoprecipitations shown in Fig. 3a and c were done using anti-HA antibody sepharose. Lysis and washing buffer was IPD1 buffer (50 mM Tris pH7.5, $50 \mathrm{mM} \mathrm{NaCl}, 2 \mathrm{mM} \mathrm{MgCl} 2,0.1 \%$ Nonidet P-40) containing $1 \mathrm{mM} \beta$-mercaptoethanol and $1 \times$ 'COMPLETE' protease inhibitor mix (Boeringer Manheim). Bound proteins were eluted with $50 \mathrm{mM}$ Tris pH7.5, $100 \mathrm{mM} \mathrm{NaCl}, 2 \mathrm{mM} \mathrm{MgCl}_{2}, 1 \%$ SDS, $1 \mathrm{mg} \mathrm{ml}^{-1}$ HA peptide and $1 \times$ COMPLETE protease inhibitor mix, and analysed for presence of Dcpl by western blotting ${ }^{23}$. The Dcp 1 immunoprecipitation shown in Fig. $3 c$ was done similarly using anti-Dcpl polyclonal antibodies and protein A sepharose, and the co-immunoprecipitating Lsm1 was revealed in western blots using anti-Lsml polyclonal antibodies. Elution buffer was as above but without HA peptide. The immunoprecipitation of FLAG-Lsm4 shown in Fig. 3a was done similarly using anti-FLAG antibody agarose (Sigma). Lysis was in IPD2 buffer $(50 \mathrm{mM}$ Tris pH7.5, $25 \mathrm{mM} \mathrm{NaCl}$, $2 \mathrm{mM} \mathrm{MgCl}_{2}, 0.2 \%$ Nonidet P-40). IPD2 buffer without Nonidet P-40 was used to wash the beads. Elution buffer was as above but with $200 \mu \mathrm{g} \mathrm{ml}^{-1}$ FLAG peptide. HA-Lsm immunoprecipitation in Fig. 3b was done using anti-HA antibodies and protein A sepharose. Lysis and washing buffer was $6 \mathrm{mM}$ HEPES pH7.9, $150 \mathrm{mM} \mathrm{NaCl}, 2.5 \mathrm{mM}$ $\mathrm{MgCl}_{2}, 0.05 \%$ Nonidet P-40. Immunoprecipitated proteins were extracted by boiling pellets in SDS sample buffer and western analysed using anti-Patl polyclonal antibodies. To study the RNA dependence of co-immunoprecipitations, lysates were treated with $0.5 \mu \mathrm{gll}^{-1}$ RNase A for $10 \mathrm{~min}$ at room temperature before preclearing. To study the co-immunoprecipitation of RNA, total RNA extracted from the immunoprecipitates was analysed by northern blotting using $\mathrm{CYH} 2$ complementary DNA probe or U6 oligo probe (oRP 759: 5'-GACCAAATGTCCACGAAGGG-3').

Received 29 July 1999; accepted 10 February 2000

. Beelman, C. A. \& Parker, R. Degradation of mRNA in eukaryotes. Cell 81, 179-183 (1995).

2. Jacobson, A. \& Peltz, S. W. Interrelationships of the pathways of mRNA decay and translation in eukaryotic cells. Аnпи. Rev. Biochem. 65, 693-739 (1996).

3. Seraphin, B. Sm and Sm-like proteins belong to a large family: identification of proteins of the $\mathrm{U} 6$ as well as the U1, U2, U4 and U5 snRNPs. EMBO J. 14, 2089-2098 (1995).

4. Hermann, H. et al. snRNP Sm proteins share two evolutionarily conserved sequence motifs which are involved in Sm protein-protein interactions. EMBO J. 14, 2076-2088 (1995).

5. Cooper, M., Johnston, L. H. \& Beggs, J. D. Identification and characterization of Usslp (Sdb23p): a novel U6 snRNA-associated protein with significant similarity to core proteins of small nuclear ribonucleoproteins. EMBO J. 14, 2066-2075 (1995).

6. Salgado-Garrido, R., Bragado-Nilsson, E., Kandels-Lewis, S. \& Séraphin, B. Sm and Sm-like protein assemble in two related complexes of deep evolutionary origin. EMBO J. 18, 3451-3462 (1999).

7. Mayes, A. E., Verdone, L., Legrain, P. \& Beggs, J. D. Characterization of Sm-like proteins in yeast and their association with U6 snRNA. EMBO J. 18, 4321-4331 (1999).

8. Pannone, B. K., Xue, D. \& Wolin, S. L. A role for the yeast La protein in U6 snRNP assembly: evidence that the La protein is a molecular chaperone for RNA polymerase III transcripts. EMBO J. 17, 7442 7453 (1998)

9. Stevens, S. W. \& Abelson, J. Purification of the yeast U4/U6.U5 small nuclear ribonucleoprotein particle and identification of its proteins. Proc. Natl Acad. Sci. USA 96, 7226-7231 (1999).

10. Boeck, R., Lapeyre, P., Brown, C. E. \& Sachs, A. B. Capped mRNA degradation intermediates accumulate in the yeast spb8-2 mutant. Mol. Cell. Biol. 18, 5062-5072 (1998).

11. Decker, C. J. \& Parker, R. A turnover pathway for both stable and unstable mRNAs in yeast: evidence for a requirement for deadenylation. Genes Dev. 7, 1632-1643 (1993).

12. Hatfield, L., Beelman, C. A., Stevens, A. \& Parker, R. Mutations in trans-acting factors that inhibit mRNA decapping in Saccharomyces cerevisiae. Mol. Cell. Biol. 16, 5830-5838 (1996).

13. He, W. \& Parker, R. Analysis of mRNA decay pathway in S. cerevisiae. Methods 17, 3-10 (1999).

14. Beelman, C. A. et al. An essential component of the decapping enzyme required for normal rates of mRNA decay in yeast. Nature 382, 642-646 (1996).

15. Dunckley, T. \& Parker, R. The DCP2 protein is required for mRNA decapping in Saccharomyces cerevisiae and contains a functional MutT motif. EMBO J. 18, 5411-5422 (1999).

16. Muhlrad, D., Decker, C. J. \& Parker, R. Deadenylation of the unstable mRNA encoded by the yeast MFA2 gene leads to decapping followed by $5^{\prime} \rightarrow 3^{\prime}$ digestion of the transcript. Genes Dev. 8, 855-866 (1994).

17. Fromont-Racine, M. et al. Interactions of the Lsm proteins of S. cerevisiae Proc. Natl Acad. Sci. USA (submitted)

18. Wang, X., Watt, P. M., Louis, E. J., Borts, R. H. \& Hickson, I. D. Pat1: a topoisomerase II-associated protein required for faithful chromosome transmission in Saccharomyces cerevisiae. Nucleic Acids Res. 24, 4791-4797 (1996)

19. Kambach, C. et al. Crystal structures of two Sm protein complexes and their implications for the assembly of the spliceosomal snRNPs. Cell 96, 375-387 (1999).

20. Schwartz, D. \&s Parker, R. Mutations in translation initiation factors lead to increased rates of deadenylation and decapping of mRNAs in Saccharomyces cerevisiae. Mol. Cell. Biol. 19, 5247-5256 (1999)
21. Achsel, T. et al. A doughnut-shaped heteromer of human Sm-like proteins binds to the $3^{\prime}$-end of U6 snRNA, thereby facilitating U4/U6 duplex formation in vitro. EMBO J. 18, 5789-5802 (1999).

22. Dix, I., Russell, C., Yehuda, S. B., Kupiec, M. \& Beggs, J. D. The identification and characterization of a novel splicing protein, Isylp, of Saccharomyces cerevisiae RNA 5, 360-368 (1999).

23. Tharun, S. \& Parker, R. Analysis of mutations in the yeast mRNA decapping enzyme. Genetics 151, 1273-1285 (1999)

\section{Acknowledgements}

We thank members of R.P.'s laboratory for useful discussions and comments on the manuscript. This study was supported by funds from the Howard Hughes Medical Institute, the NIH, a Wellcome Trust Prize Studentship to A.E.M. and a Royal Society Cephalosporin Fund Senior Research Fellowship to J.D.B. We also thank N. RodriquezCousino for the antisera to Pat l, C. Russell for providing the HA-lsyl strain and A. Sachs for the anti-Lsm1 antibodies.

Correspondence and requests for materials should be addressed to R.P. (e-mail: rrparker@u.arizona.edu).

\section{Structural basis for the anticoagulant activity of the thrombin- thrombomodulin complex}

\section{Pablo Fuentes-Prior*, Yoriko Iwanaga*, Robert Huber*, Rene Pagila $\dagger$, Galina Rumennik $\dagger$, Marian Seto $\dagger$, John Morser $\dagger$, David R. Light $\dagger$ \&. Wolfram Bode ${ }^{x}$}

* Max-Planck-Institut für Biochemie, Abteilung Strukturforschung Am Klopferspitz 18a, D-82152 Martinsried, Germany

$\dagger$ Berlex Biosciences, Richmond, California 94804, USA

The serine proteinase $\alpha$-thrombin causes blood clotting through proteolytic cleavage of fibrinogen and protease-activated receptors and amplifies its own generation by activating the essential clotting factors V and VIII ${ }^{1}$. Thrombomodulin ${ }^{2}$, a transmembrane thrombin receptor with six contiguous epidermal growth factorlike domains (TME1-6), profoundly alters the substrate specificity of thrombin from pro- to anticoagulant by activating protein $\mathrm{C}$ (see, for example, reference 2). Activated protein $\mathrm{C}$ then deactivates the coagulation cascade by degrading activated factors $\mathrm{V}$ and VIII $^{2}$. The thrombin-thrombomodulin complex inhibits fibrinolysis by activating the procarboxypeptidase thrombinactivatable fibrinolysis inhibitor ${ }^{3}$. Here we present the $2.3 \AA$ crystal structure of human $\alpha$-thrombin bound to the smallest thrombomodulin fragment required for full protein- $\mathrm{C}$ co-factor activity, TME456. The Y-shaped thrombomodulin fragment binds to thrombin's anion-binding exosite-I, preventing binding of procoagulant substrates. Thrombomodulin binding does not seem to induce marked allosteric structural rearrangements at the thrombin active site. Rather, docking of a protein $\mathrm{C}$ model to thrombin-TME456 indicates that TME45 may bind substrates in such a manner that their zymogen-activation cleavage sites are presented optimally to the unaltered thrombin active site.

Thrombomodulin consists of a lectin-like amino-terminal domain, followed by a hydrophobic segment, six contiguous epidermal growth factor (EGF)-like domains, an $\mathrm{O}$-glycosylated serine/threonine-rich domain, a transmembrane segment and a short cytoplasmic tail (Fig. 1a). Upon thrombomodulin binding, thrombin activation of both protein $\mathrm{C}$ and thrombin-activatable fibrinolysis inhibitor (TAFI) is enhanced by at least three orders of magnitude ${ }^{2,3}$. Different hypotheses have been proposed to explain the thrombomodulin-induced switch in thrombin specificity. Mutagenesis studies (see, for example, ref. 4) and experiments with fluorescence $e^{5}$ or spin label ${ }^{6}$ active-site probes indicate that thrombomodulin binding may cause allosteric changes in the 